\title{
DRAVET SYNDROME AS ADULT FORM OF EPILEPTIC ENCEPHALOPATHY
}

Researchers at the National Hospital, Queen Square, Great Ormond Street Hospital, and other centers in the UK and Melbourne, Australia identified a series of 22 adult patients (median age 39 years; range 20-66), including 3 post-mortem cases with Dravet syndrome. SCN1A mutations were found in $12(60 \%)$ of 20 adult patients, novel in 11. A family history of epilepsy and/or febrile seizures was present in $9(41 \%)$ adult patients. Seizure onset in infancy was triggered by vaccination in $11(41 \%)$ cases, pertussis or triple vaccine. Autistic behavior developed in childhood in 15 (68\%) cases. Clinical features of Dravet syndrome in adults included multiple seizure types despite polytherapy, fever sensitivity (slight variation in temperature with infection triggered seizure in $9(41 \%)$ cases), and cognitive and motor deterioration. SCN IA mutations were found in $12(60 \%)$ of 20 adult cases. Dysphagia developing after the fourth decade caused significant morbidity, or death. Death in 4 adult cases was due to bronchopneumonia in 3 and sudden unexplained death in 1. None died of convulsive status epilepticus. MRI was mostly normal or showed nonspecific cerebral and cerebellar atrophy. Interictal EEG showed slow background activity and focal or multifocal epileptiform discharges. Ictal discharges were focal and none were generalized. Nonconvulsive status epilepticus was shown on video-EEG in 2 patients with subtle seizures, previously confused with behavior problems. When correctly diagnosed at a later age, treatment changes improved seizure control, with significant improvement in cognitive performance and quality of life in 2 cases. Anticonvulsants previously reported to worsen seizures in Dravet syndrome, including lamotrigine, carbamazepine, vigabatrin, phenytoin, or oxcarbazepine, were sometimes replaced with valproate or levetiracetam. Neuropathological examinations revealed no characteristic abnormalities; preservation of the neocortex and hippocampal neurons and interneurons was remarkable, despite decades of poorly controlled seizures. Dravet syndrome is considered a "syndrome carrying higher risk of epileptic encephalopathy" in the recent reorganization of the International League Against Epilepsy classification (Berg et al. 2010; Engel et al. 2001), and the study supports this definition. (Catarino CB, Liu JYW, Liagkouras I, et al. Dravet syndrome as epileptic encephalopathy: evidence from long-term course and neuropathology. Brain 2011;134:2982-3010). (Respond: Dr Sanjay M Sisodiya, Dept Clinical and Experimental Epilepsy, National Hospital for Neurology and Neurosurgery, Queen Square, London WC1N 3BG, UK. E-mail:s.sisodiya@ion.ucl.ac.uk).

COMMENT. Testing for SCN1A mutations and the diagnosis of Dravet syndrome should be considered in adults with a long history of seizures resistant to anticonvulsant therapy and fever sensitivity. A positive diagnosis could lead to improved seizure control and quality of life. The absence of neuropathological evidence of brain cell degeneration, despite years of uncontrolled seizures, is remarkable. Other noteworthy features of the adult Dravet patients included the high incidence of seizure onset in infancy attributed to a vaccination trigger, and the persistence of fever sensitivity and a low febrile seizure threshold past the defined 5-year limit for febrile seizures. 